weise kommt es naturgemäß weniger an und man muß dem Verfasser unbedingt zustimmen, wenn er sagt: ,Solange man sich nicht die Vektoroperationen ihrem Wesen nach vorstellt, so lange man nicht mit Vektoren rechnet, ist die Bezeichnungsweise keinen Pfennig wert." Das Buch behandelt die elementaren Operationen der Vektoranalysis, die Differentialoperationen und Integralsätze der Vektoranalysis; krummlinige Koordinaten, Vektorzerlegungen, mechanische Deformationen, sowie Anwendungen aus der Hydrodynamik und aus der Elektrodynamik.

St. $M$.

\title{
Theorie der Elektrizität. Zweiter Band: Elektromagnetische
} Theorie der Strahlung von. Dr. M. Abraham. Mit 5 Textfig., 404 S. $8^{0}$. Verlag von B. G. Teabner, Leipzig, 1905.

Über den ersten Band dieses Werkes wurde XVI., S. 68, berichtet. Im. vorliegenden Buche werden die "Konvektionsstrahlung" und die "Wellenstrahlung" behandelt. Der erste Abschnitt: Das Feld and die Bewegung der einzelnen Elektronen führt zunächst die Tatsachen auf, welche die Annahme einer atomistischen Struktur der Elektrizität nahelegen. Aach werden die allgemeinen Lösungen der Grundgleichungen mit Hilfe dex elektromagnetischen Potentiale gegeben. Es folgt die Thorie der Wellenstrahlung einer bewegten Punktladung and die Mechanik der Elektronen, wobei an der Annahme eines starren kugelförmigen Elektrons festgehalten wird.

Der zweite Abschnitt: Elektromagnetische Vorgänge in wägbaren Körpern enthält die Hauptgleichungen für ruhende (Maxwell) und bewegte Körper (Lorentz). Es werden hier auch einige Probleme behandelt, die mit der atomistischen Hypothese nor lose zusammenhängen. Auch die drahtlose Telegraphie und die Antennentheorie haben Platz gefunden. Spezielles Interesse widmet der Verf. dem Problem der Reflexion des Lichtes an einem bewegten Spiegel. Die Lösung ist innigst mit dem thermodynamischen Gesetz der strahlenden Wärme verknüpft. Anch anf die Schwierigkeiten, die der Elektronentheorie aus dem Mangel eines nachweisbaren Einflasses der Erdbewegung auf das Licht irdischer Lichtquellen erwachsen, ist hingewiesen. Das Werk, das einen Rückblick auf den gegenwärtigen Stand der Forschung geben soll, wird nicht verfehlen, wesentlich zur Klärung der Begriffe beizutragen.

St. $M$.

Annuaire du Bureau des Longitudes pour 1906. Paris, Gauthier-Villars. $16^{0}, 900$ pag., 1 Fres. 50.

Das neue Jahrbuch enthält nach dem neuen Plan außer dem astronomischen Teil vorwiegend physikalische und chemische Daten, dagegen sind die Angaben aus Geographie und Statistik für 1907 zurückgestellt, so daß ron nun an diese Gebiete abwechselnd im Annuaire zur Geltung kommen sollen. İm astronomischen Teil sind die barometrische Höhenbestimmung, die Fixsternparallaxen, die Eigenbewegung and die Spektroskopie unterdrückt, dafür aber sind die Elemente der kleineren Planeten vollständig aufgenommen. Auch das Verzeichnis der veränderlichen Sterne ist kompliziert. Im chemischen Teil sind die thermochemischen Angaben wieder publiziert, welche seit einiger Zeit zurückgestellt werden maßten. 